\title{
A randomized controlled trial to study the efficacy of intravaginal hormonal ring for control of heavy menstrual bleeding as compared to combined oral contraceptive pills
}

\author{
Tarnima Saha1, Meenakshi K. Bharadwaj $^{1 *}$, Shakti Vardhan ${ }^{2}$
}

\begin{abstract}
${ }^{1}$ Department of Obstetrics and Gynecology, Armed Forces Medical College, Pune, Maharashtra, India
${ }^{2}$ Department of Obstetrics and Gynecology, Military Hospital, Devlali, Nashik, Maharashtra, India
\end{abstract}

Received: 08 January 2020

Accepted: 05 February 2020

*Correspondence:

Dr. Meenakshi K. Bharadwaj,

E-mail: mkb.doc@gmail.com

Copyright: (c) the author(s), publisher and licensee Medip Academy. This is an open-access article distributed under the terms of the Creative Commons Attribution Non-Commercial License, which permits unrestricted non-commercial use, distribution, and reproduction in any medium, provided the original work is properly cited.

\begin{abstract}
Background: Heavy menstrual bleeding (HMB) is one of the commonest presenting complaints in reproductive age group. Although combined oral contraceptives (COCs) are commonly used in such patients, combined hormones by intravaginal route has been found acceptable and effective. Aim of the study is to compare the efficacy and side effects of combined intravaginal hormonal ring (IHR) with COCs in control of HMB in these patients.

Methods: Hundred women with HMB fulfilling inclusion criteria were randomized into two equal groups and treated with either IHR or COCs for three cycles. Each cycle consisted of three weeks of IHR/COC use followed by 1 -week ring-free/non-hormonal pills period. Outcome measures were change in PBAC score (pictorial blood loss assessment chart), hemoglobin rise, side effects and overall patient satisfaction.

Results: The percentage reduction in PBAC score, the duration of menses and increase in hemoglobin levels were statistically significant at the end of study in each group. The PBAC score reduction was $87.37 \%$ vs $61.52 \%$, menses duration was $4.24 \pm 0.74$ versus 5.16 \pm 1.67 , and hemoglobin increase was 3.16 (95\% CI:0.142-1.412) and 1.24 (95\% CI:1.048-1.640) in the IHR versus COC group. However, the intergroup reduction of mean PBAC score was not statistically significant. Significantly more ring users were satisfied and elected to continue with treatment.

Conclusions: Both the IHR and COCs are effective treatments for HMB in reproductive age group. IHR may be an attractive option for HMB due to better compliance and lesser systemic side-effects.
\end{abstract}

Keywords: Combined oral contraceptive pills, Heavy menstrual bleeding, Intravaginal hormonal ring, Pictorial blood loss assessment chart

\section{INTRODUCTION}

Heavy menstrual bleeding (HMB) is the single most common complaint that reproductive women present with to their clinicians. Though exact incidence is unknown, it is a common gynaecological problem. HMB is defined as prolonged or heavy cyclical menstruation lasting longer than 7 days or exceeding $80 \mathrm{ml}$ blood loss in each cycle. (ACOG Practice Bulletin 2012). ${ }^{1}$ The National Institute of Clinical Excellence in the UK (NICE Guidelines) defines HMB as "excessive menstrual blood loss that interferes with the woman's physical, emotional, social and/or material quality of life". ${ }^{2}$ HMB is a descriptive nomenclature of abnormal uterine bleeding (AUB), which is defined as bleeding from the uterine corpus that is abnormal in regularity, volume, frequency, or duration and occurs in the absence of pregnancy. ${ }^{1}$ Because of inherent difficulties of objective menstrual blood loss measurement, visual scoring based on pictorial blood loss assessment chart developed by Higham et al. has been used as the main determinant of HMB commonly. ${ }^{3}$ Janssen et al, reported predictive values of 
positive and negative test of $85.9 \%$ and $84.8 \%$ respectively, using a PBAC score of 185 as the cut-off point. ${ }^{4}$

Eighty percent of women treated for HMB have no structural pathology, which makes medical management the preferred initial option of treatment and possibly avoids unnecessary surgery. ${ }^{5}$ Medical treatment options for $\mathrm{HMB}$ include prostaglandin synthetase inhibitors, antifibrinolytics, COC pills, oral progestogens, GnRH analogues, levonorgestrel releasing intrauterine system (LNG-IUS) and hormone releasing vaginal rings. Surgery should be reserved only when medical treatment fails. Surgical options include conservative surgery (hysteroscopy with dilatation and curettage, polypectomy, myomectomy or endometrial ablative techniques) and hysterectomy.

COCs containing oestrogen and progesterone is an established hormonal treatment in HMB. It decreases the blood loss due to haemostatic effect of estrogen and also regularizes the cycle. ${ }^{6}$ Treatment of HMB has evolved rapidly in last few years. Recently new hormones in lower doses and other routes of administration are being studied to reduce hormone-related side-effects and improve patient compliance. Earlier only oral route was available. Oral treatment fails because of poor compliance and systemic side-effects. Hence other routes of treatment became available; with new technology, other options like parenteral, subdermal, nasal, and intravaginal routes came into existence.

Intravaginal route has been found to be effective and acceptable. Vaginal administration of hormonal ring has several advantages such as gastrointestinal absorption and hepatic first-pass metabolism are avoided, and hormones are continuously absorbed by the vaginal mucosa by controlled release formulation producing steady, uniform serum hormone levels. The convenience of once-a-month administration is another major advantage. Oestrogen and progesterone are released at lower doses than oral pills with uterus receiving the required dose and lesser adverse effects of the hormones. Others potential benefits are easy insertion, removal and self-control by user, no daily intervention and return of cyclical menses after removal, hence better user compliance. It is more discreet to use and has less breakthrough bleeding or spotting as compared to COCs. Various studies have been done in past to demonstrate contraceptive efficacy of NuvaRing and found it comparable to oral pills. ${ }^{7-9}$ However, efficacy of combined hormonal vaginal ring in HMB has not been assessed well, hence the study was conducted to compare the efficacy and safety of vaginal ring versus COC pills in medical management of HMB.

\section{METHODS}

This was a prospective, randomized controlled trial recruiting women with heavy menstrual bleeding presenting to the outpatient departments of gynecology, Armed Forces Medical College, Pune and Command
Hospital (Southern Command), Pune in Maharashtra, India in the period from January 2018 to July 2019. Ethical clearance was taken from the Institutional Ethical Committee in October 2017.

The study protocol was registered at the Clinical Trials Registry- India (CTRI) in January 2018 at www.ctri.nic.in (ID: CTRI/2018/01/011586) in accordance with the Declaration of Helsinki and the recommendations of The Committee of Editors of Biomedical Journals of 11 major journals of India, as has been made mandatory by the Drugs Controller General India (DGCI).

\section{Inclusion criteria}

All women in the age group 18-45 years having heavy menstrual bleeding due to

- $\quad$ AUB-O

- $\quad$ AUB-E and/or

- AUB-L (fibroid <2 cm), as per PALM-COEIN classification.

\section{Exclusion criteria}

Women having heavy menstrual bleeding due to

- AUB-Polyp

- AUB-Adenomyosis

- AUB-Leiomyoma (fibroids $>2 \mathrm{~cm}$ )

- AUB-Malignancy or hyperplasia

- AUB-Coagulopathy

- AUB-Iatrogenic

- AUB-Not yet classified.

- Medical conditions where COC pills/IHR are contraindicated like jaundice, diabetes mellitus, hypertension, migraine, epilepsy, h/o thromboembolism, breast or genital tract malignancies, genital prolapse or infections, stroke and smokers

- Any hormonal therapy, anticoagulants and/or antifibrinolytics in the last 6 months

- Patients unwilling for contraception and medical management.

A detailed menstrual, obstetric and medical history was taken and recorded in the case record proforma. General and gynaecological examinations were carried out. Baseline weight and blood pressure were recorded. Hemogram, prothrombin time, INR, thyroid function test, ultrasound evaluation, PAP smear and endometrial biopsy were performed in all cases. Hysteroscopy was performed in selected case, e.g., endometrial polyps and submucous fibroid.

\section{Consent and randomisation}

A total of 157 women were screened for the study and 57 women who did not meet the inclusion criteria or had not consented to participate in the study were excluded from 
it. Of the 100 women remaining, randomisation was done with the help of computer-generated random number table into two groups: Group $\mathrm{A}(\mathrm{n}=50)$ wherein patients were instructed to use intravaginal hormonal ring (NuvaRing) and Group B (n=50) in which low dose COC pills (MALA-N) were given (Figure 1). A written informed consent was obtained from each participant (fulfilling inclusion criteria) after detailed information about the purpose and nature of the study. There was no blinding of the patients or the investigator because of the different nature of treatments. However, outcome assessors, that is, those performing laboratory investigations and statistical analysis, were blinded to the treatment groups.

\section{Consort flowchart}

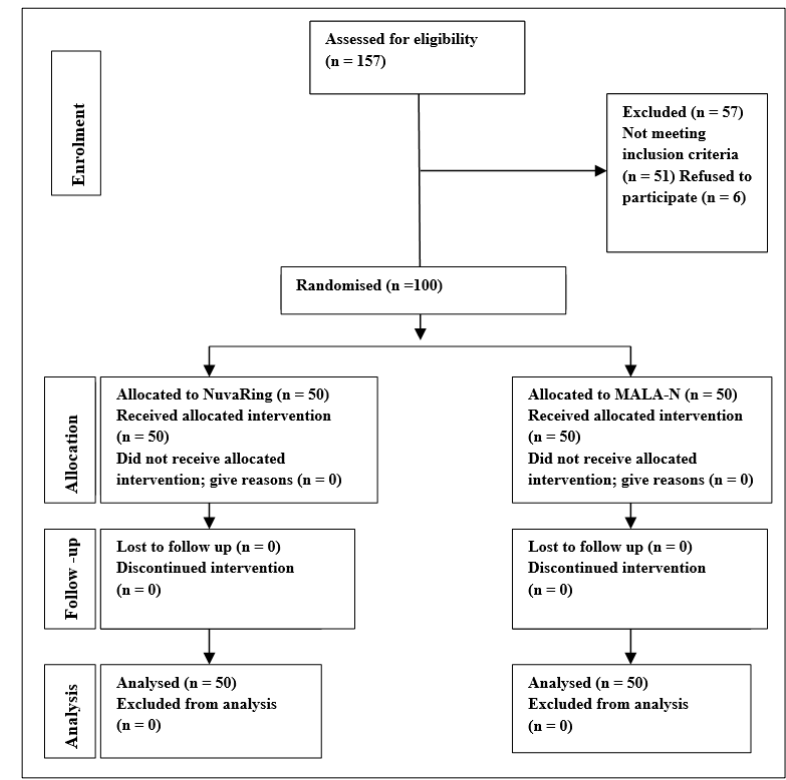

Figure 1: CONSORT flowchart of participants in the trial.

\section{Protocol and treatment}

In Group A (IHR-NuvaRing), women received verbal and written instructions on the use of the ring, including how and when they should insert and remove it. For the first cycle, the ring was inserted between days 4 and 6 of the menstrual cycle, and removed after three weeks of insertion, i.e. each cycle consisted of 3 weeks of ring use followed by a 1 -week ring-free period during which there was withdrawal bleeding. Each NuvaRing, (a combined contraceptive hormonal ring, manufactured by Merck, for once-a-month intravaginal application) contains ethinylestradiol (EE, $2.7 \mathrm{mg}$ ) releasing $15 \mu \mathrm{g}$ and etonogestrel (ENG, $11.7 \mathrm{mg}$ ) releasing $120 \mu \mathrm{g}$ daily and intended for single use of 21 days. A new ring was reinserted after treatment-free period of one week. Treatment continued for three cycles. Similarly, in Group B, low-dose COC tablets, MALA-N (available in government supply, manufactured by Hindustan Latex
Ltd.) containing $30 \mu \mathrm{g}$ EE and $150 \mu \mathrm{g}$ levonorgestrel were administered starting from day 4 to day 6 of menstrual cycle for 21 days followed by non-hormonal (iron) tablets for next 7 days during which there was withdrawal bleeding. A new pack was started immediately after completion of pack of 28 tablets. Treatment was continued for three cycles in this arm also.

Women in both groups were followed up monthly during the treatment period when PBAC score, duration of bleeding and any adverse effects were noted to assess the patients' response to treatment. Patients were instructed to evaluate the degree of saturation of each sanitary pad and/or tampon used, any episodes of flooding as well as passage of clots during menstruation. Total numbers of points were tallied for each menses. To increase the reliability of the measurements, the participants were instructed on how to complete the PBAC, and all participants completed one cycle during the screening phase of the study. Blood was taken at the beginning and end of the study to measure haemoglobin levels.

\section{Outcome measures}

Primary outcome measure was change in PBAC score. Secondary outcomes included side effects, change in haemoglobin and weight from baseline to three cycles after treatment.

\section{Statistical analysis}

Data obtained were statistically analyzed using the Statistical Package for Social Sciences (SPSS 20) software version 20.0 for Windows. All analyses were intention to treat. Quantitative variables were summarized as mean and SD. Qualitative variables were summarized as proportion. Means were compared between the two study groups using the unpaired Student's t test, while proportions were compared using the $\chi 2$ test. Baseline demography and clinical profile were analyzed using unpaired student t-test. Comparison inside each group was based on the change in mean using a paired t test for continuous variables and the McNemar test for categorical variables. A p value of less than .05 was considered statistically significant.

\section{RESULTS}

A hundred women fulfilling inclusion criteria were randomly assigned to either the IHR (Group A; $n=50$ ) or COC (Group B; n=50) group. All participants completed three cycles of treatment. The baseline characteristics between both groups were compared and no significant statistical differences were observed in them (Table 1). The mean PBAC score at entry to the study was $624.86 \pm 391.71$ and $515 \pm 238.9$ in the IHR and COC groups respectively. The mean PBAC score decreased significantly in both IHR and COC group at the end of the 3 cycles $(78.92 \pm 53.91$ versus $198.16 \pm 142.79)$ (Table $2)$. 
Table 1: Patient characteristics.

\begin{tabular}{|llll|}
\hline & Group A (IHR; $\mathbf{n = 5 0 )}$ & Group B $(\mathbf{C O C} ; \mathbf{n = 5 0})$ & p value \\
\hline Age (years) & $38.1 \pm 5.35$ & $36.6 \pm 7.89$ & 0.38 \\
\hline Parity & & $2(4 \%)$ & 1.00 \\
\hline 0 & $2(4 \%)$ & $9(18 \%)$ & 0.57 \\
\hline 1 & $6(12 \%)$ & $25(50 \%)$ & 1.00 \\
\hline 2 & $25(50 \%)$ & $14(28 \%)$ & 0.68 \\
\hline$\geq 3$ & $17(34 \%)$ & $55.64 \pm 7.91$ & 0.83 \\
\hline Weight $(\mathrm{kg})$ & $55.68 \pm 10.89$ & $25.4 \pm 3.2$ & 0.39 \\
\hline BMI $\left(\mathrm{kg} / \mathrm{m}^{2}\right)$ & $24.8 \pm 3.8$ & $27.84 \pm 8.72$ & 0.92 \\
\hline Cycle length & $29.18 \pm 11.45$ & $8.6 \pm 3.31$ & 0.42 \\
\hline Duration of menses (days) & $10.1 \pm 6.95$ & $515 \pm 238.98$ & 0.11 \\
\hline PBAC score $($ baseline $)$ & $624.86 \pm 391.7$ & $11.13 \pm 1.68$ & 0.84 \\
\hline Haemoglobin $(\mathrm{g} / \mathrm{dl})$ & $10.25 \pm 1.64$ & & \\
\hline Data are shown as $\mathrm{n}(\%)$ or mean + SD. None of the differences were statistically significant $(\mathrm{p}<0.05)$ & \\
\hline
\end{tabular}

Data are shown as $\mathrm{n}(\%)$ or mean \pm SD. None of the differences were statistically significant $(\mathrm{p}<0.05)$.

Table 2: Effect of therapy in each group (Mean \pm SD).

\begin{tabular}{|lllllll|}
\hline \multicolumn{4}{c}{ Group A (IHR) } & & \multicolumn{3}{l|}{ Group B (COC) } & \\
\hline Baseline & 3 months & p value & Baseline & 3 months & p value \\
\hline Cycle length (days) & $29.86 \pm 391.71$ & $78.92 \pm 53.91$ & $0.0499^{*}$ & $515 \pm 238.98$ & $198.16 \pm 142.79$ & $0.0003^{*}$ \\
\hline $\begin{array}{l}\text { Duration of } \\
\text { menses (days) }\end{array}$ & $10.1 \pm 6.95$ & 28 & 0.488 & $27.84 \pm 8.72$ & 28 & 0.702 \\
\hline Haemoglobin (g/dl) & $10.26 \pm 1.64$ & $13.42 \pm 0.71$ & $0.0174^{*}$ & $11.13 \pm 1.68$ & $12.37 \pm 0.99$ & $0.000000000004^{*}$ \\
\hline
\end{tabular}

Means were compared inside each group using the paired student's t test, *Statistically significant difference: $\mathrm{p}<0.05$.

Table 3: Comparison of therapy in both groups at the end of the study (cycle 3).

\begin{tabular}{|llll|}
\hline & Group A (IHR; $\mathbf{n = 5 0})$ & Group B $(\mathbf{C O C} ; \mathbf{n = 5 0})$ & p value \\
\hline PBAC Score & $\mathbf{7 8 . 9 2 \pm 5 3 . 9 1}$ & $\mathbf{1 9 8 . 1 6 \pm 1 4 2 . 7 9}$ & 0.95 \\
\hline Percentage reduction in PBAC score & $87.37 \%$ & $61.52 \%$ & $0.001^{*}$ \\
\hline Cycle length (days) & 28 & 28 & - \\
\hline Duration of menses & $4.24 \pm 0.74$ & $5.16 \pm 1.67$ & 0.64 \\
\hline Haemoglobin & $13.42 \pm 0.71$ & $12.37 \pm 0.99$ & 0.34 \\
\hline
\end{tabular}

Data are shown as mean \pm S.D., unless otherwise indicated. None of the differences were statistically significant $(p<0.05)$, except percentage reduction in PBAC score.

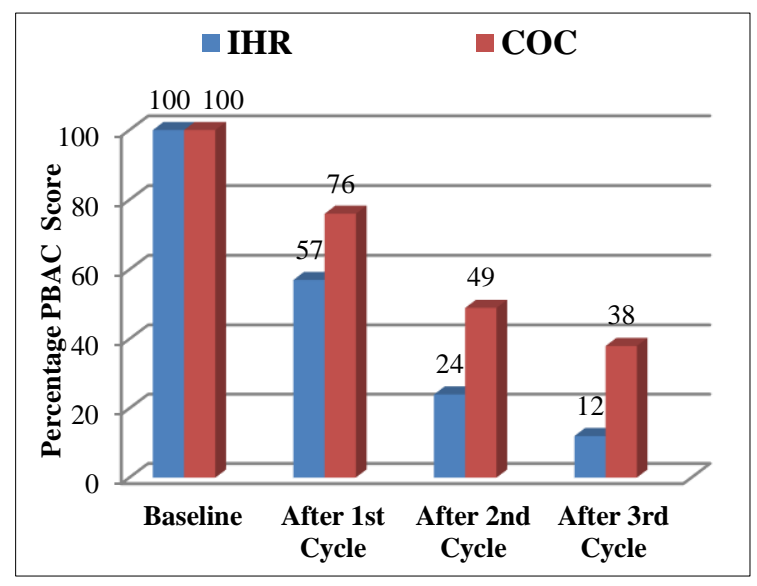

Figure 2: Comparison of effect of therapy on percentage reduction in blood loss/PBAC score in both the groups during treatment.

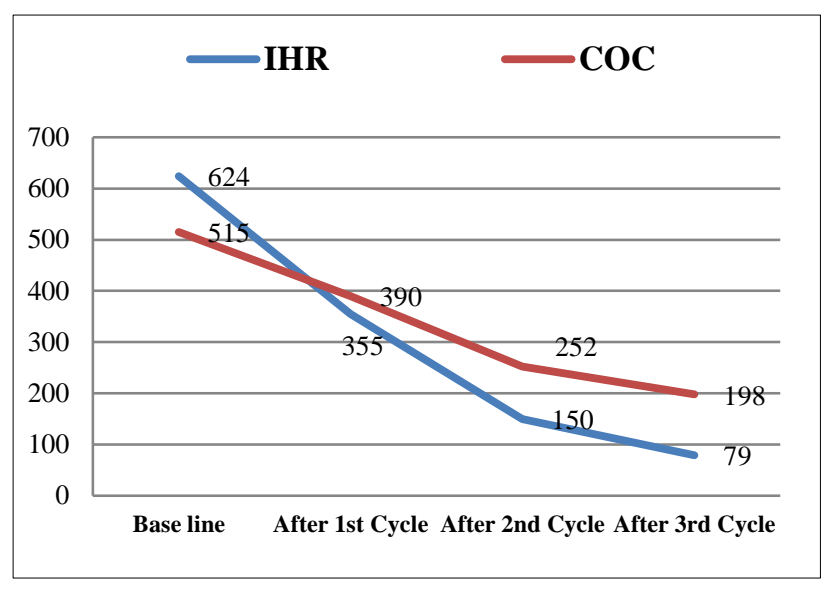

Figure 3: Effect of therapy on PBAC score in both groups at 3 follow-up visits during treatment. 
This decrease was seen more in the IHR group, however the intergroup difference in this decrease was not statistically significant (Table 3). The percentage reduction of blood loss calculated as [(end-of-study PBAC score - baseline PBAC score/ baseline PBAC score $) \times 100](87.37 \%$ in the IHR group versus $61.52 \%$ in the COC group) was also detected to be statistically significant (Figure 2). The decreasing trend in PBAC score was registered after each cycle of use of IHR and $\mathrm{COC}$ in the follow-up visits during treatment (Figure 3 ). There was a reduction in duration of menstruation in both the groups at the end of the study but the comparison of intergroup reduction in it was not statistically significant. Due to the decrease in blood loss during menstruation the haemoglobin levels improved significantly in patients in both the groups at the end of the 3 cycle (Table 4).

Table 4: Effect of combined hormonal drugs on haemoglobin and weight.

\begin{tabular}{|llllll|} 
Variable & Group & Pre-therapy & Post-therapy & Difference (pre-post) $(\mathbf{9 5 \%}$ CI) & p value \\
\hline $\begin{array}{l}\text { Haemoglobin } \\
\text { (g/dl) }\end{array}$ & IHR & $10.26 \pm 1.64$ & $13.42 \pm 0.71$ & $-3.16(0.142,1.412)$ & $0.0174^{*}$ \\
\cline { 2 - 6 } & COC & $11.13 \pm 1.68$ & $12.37 \pm 0.99$ & $-1.24(1.048,1.640)$ & $0.000000000004^{*}$ \\
\hline $\begin{array}{l}\text { Weight } \\
\text { (kg) }\end{array}$ & IHR & $55.66 \pm 10.89$ & $55.75 \pm 10.89$ & $-0.09(0.979,1.015)$ & NS \\
\cline { 2 - 6 } & COC & $55.64 \pm 7.91$ & $56.20 \pm 7.93$ & $-0.56(0.954,1.026)$ & NS \\
\hline
\end{tabular}

Data are shown as mean \pm SD, $*$ Statistically significant difference, $p<0.05$, NS: Difference not statistically significant.

Table 5: Side effects and overall patients' satisfaction with treatment.

\begin{tabular}{|llll|}
\hline & Group A $($ IHR; $\mathbf{n = 5 0})$ & Group B $(\mathbf{C O C} ; \mathbf{n = 5 0})$ & p value \\
\hline Nausea & $0(0 \%)$ & $2(4 \%)$ & 0.153 \\
\hline Headache & $0(0 \%)$ & $2(4 \%)$ & 0.153 \\
\hline Mood swings/ depression & $0(0 \%)$ & $4(8 \%)$ & $0.041^{*}$ \\
\hline Mastalgia & $1(2 \%)$ & $3(6 \%)$ & 0.31 \\
\hline Breakthrough bleeding/spotting & $2(4 \%)$ & $5(10 \%)$ & 0.238 \\
\hline Leucorrhoea & $3(6 \%)$ & $1(2 \%)$ & 0.307 \\
\hline Vaginal discomfort/vaginitis & $0(0 \%)$ & $0(0 \%)$ & No difference \\
\hline Ring expulsion & $2(4 \%)$ & - & - \\
\hline Coital difficulties & $0(0 \%)$ & $0(0 \%)$ & No difference \\
\hline Overall satisfaction with treatment & $49(98 \%)$ & $21(42 \%)$ & $0.0001^{*}$ \\
\hline Continuation with treatment & $49(98 \%)$ & $12(24 \%)$ & $0.001^{*}$ \\
\hline
\end{tabular}

Data are shown as $\mathrm{n}(\%)$ of women, $*$ Statistically significant difference: $\mathrm{p}<0.05$.

Table 5 summarizes the side effects and overall patient satisfaction with treatment. When the side-effects were compared between the two groups, mood swings/ depression was seen only in COC group, whereas none were seen in IHR group with the difference found to be statistically significant ( $p$ value $=0.041$ ). Similarly, nausea and headache were observed in only COC group, but it was not statistically significant. Mastalgia and breakthrough bleeding occurred in both the groups but were less frequent in patients using IHR. Leukorrhea was the only common complication which was reported more frequently in the IHR group. None complained of any foreign body sensation or coital difficulties because of the vaginal ring. In the study, ring expulsion occurred in 2 patients (i.e.4\%), however no patient discontinued treatment due to these additional ring-related events. There was increase in weight in consequence to the hormonal treatment in both the groups, but the difference was not found to be significant (Table 4).

At the end of this 3-month study, based on four parameters (a) reduction of PBAC score, (b) reduction in the duration of menstruation (c) regularisation of cycles and (d) fewer incidence of side effects, significantly more ring users were satisfied with treatment than COC users (98\% versus $42 \%$, respectively), and more women elected to continue the treatment with IHR than with COC (98\% versus $24 \%$, respectively).

\section{DISCUSSION}

The choice of treatment of HMB depends on important factors like clinical stability of the patient, overall acuity of the complaint, suspected cause of bleeding, desire for future fertility, and associated medical problems. Medical management being the initial line of therapy for HMB, COC causes a gradual but progressive decrease in the volume, duration of flow and dysmenorrhea by at least $60 \%$. They act by inducing a regular shedding of a thinner endometrium and inhibiting ovulation hence providing for contraception also. ${ }^{7-9}$ IHR has the same contraceptive efficacy as COCs but also offers superior cycle control with only half the dose and systemic exposure to EE. ${ }^{10-12}$ 
In this randomized controlled trial, we have evaluated the efficacy of the IHR in the management of HMB caused by AUB (O), AUB (E) and AUB ( $\mathrm{L}<2 \mathrm{~cm})$ by comparing it with COCs for three cycles using PBAC score to quantify menstrual blood loss. On literature search we could find only four studies which evaluated the role of IHR in comparison to oral hormones for treatment of HMB. Hashim A et al, compared IHR with norethisterone in 95 patients $(\mathrm{IHR}=48, \mathrm{OC}=47)$ for 3 cycles in a randomized trial. ${ }^{13}$ Agarwal $\mathrm{N}$ et al, did a randomized trial comparing ultra-low dose COC (Femilon containing $20 \mu \mathrm{g}$ EE and $120 \mu \mathrm{g}$ Desogestrel) and IHR in 50 patients (25 in each arm) in the management of heavy menstrual bleeding for 9 cycles. ${ }^{14}$ Dahiya $\mathrm{P}$ et al, compared in a randomized trial, IHR with low dose COC (MALA-N containing $30 \mu \mathrm{g} \mathrm{EE}$ and $150 \mu \mathrm{g}$ levonorgestrel) for 6 cycles. ${ }^{15}$ Another randomized trial by Jain $\mathrm{S}$. et al. compared IHR with low dose COC (MALA-N containing $30 \mu \mathrm{g}$ EE and $150 \mu \mathrm{g} \mathrm{LNG}$ ) in 60 patients (30 in each arm) for 3 cycles. ${ }^{16}$ In our study, we compared IHR with low dose COC (MALA-N) for 3 cycles in 100 patients (50 in each arm).

In comparison to all these above studies the present study had the highest baseline PBAC score (Table 6). The duration of menstruation, baseline hemoglobin and weight of the patients at entry to the study was comparable to all.

Table 6: Comparison of baseline patient characteristics with previous studies.

\begin{tabular}{|lllllll|}
\hline \multirow{2}{*}{ Study } & PBAC score & \multicolumn{2}{l}{ Haemoglobin (g/dl) } & Weight (kg) & COC \\
\hline Hashim A et al & IHR & COC & IHR & COC & IHR & - \\
\hline Agarwal N et al & 440.6 & 302.4 & 10.5 & 10.7 & - & 55.7 \\
\hline Dahiya P et al & 327.87 & 452.2 & 10 & 10.3 & 57.9 & 62.67 \\
\hline Jain S et al & 214.87 & 237.36 & - & - & 62.4 & 57.55 \\
\hline Our study & 624.86 & 515 & 10.77 & 10.15 & 56.37 & 55.64 \\
\hline
\end{tabular}

Table 7: Comparison of change in PBAC score and their percentage reduction with previous studies.

\begin{tabular}{|lllll|}
\hline \multirow{2}{*}{ Study year } & \multicolumn{2}{l|}{ End of study PBAC score } & \multicolumn{2}{l|}{ Percentage reduction in PBAC score } \\
\hline Hashim A et al & IHR & COC & IHR & COC \\
\hline Agarwal N et al & 90.2 & 92.3 & $68.66 \%$ & $69.4 \%$ \\
\hline Dahiya P et al & 178 & 204.6 & $66 \%$ & $59 \%$ \\
\hline Jain S et al & 96.96 & 99.32 & $70.73 \%$ & $70.02 \%$ \\
\hline Our study & 87 & 74.75 & $59.5 \%$ & $68.53 \%$ \\
\hline
\end{tabular}

The PBAC score was found to significantly reduce in both IHR and oral hormones groups at the end of all the studies. In the present study, the IHR group had a reduction of mean PBAC score from 624.86 to 78.92 from baseline to end, which was statistically significant ( $p$ value $=0.0499$ ), while the COC group had a reduction of mean PBAC score from 515 to 198.16 From baseline to end of study, which was again noted to be statistically significant ( $\mathrm{p}$ value $=0.0003$ ). The reduction was found to be more in the IHR group. However, the intergroup difference in reduction of PBAC score was not statistically significant in all the studies comparing IHR with oral hormones for HMB.

In this study, authors identified $87.37 \%$ reduction in PBAC score after three cycles of the IHR use which is higher compared to the four other studies, while in COC arm the results are comparable (Table 7). ${ }^{13-16}$ This may be attributed to the higher baseline PBAC score in this study. Also, the intergroup difference in percentage reduction of PBAC score was statistically in our study which was not seen in the other similar studies (Figure 4). ${ }^{13-16}$
This may be attributed to the higher baseline PBAC score in our study. Also, the intergroup difference in percentage reduction of PBAC score was statistically in our study which was not seen in the other similar studies (Figure 4)..$^{13-16}$

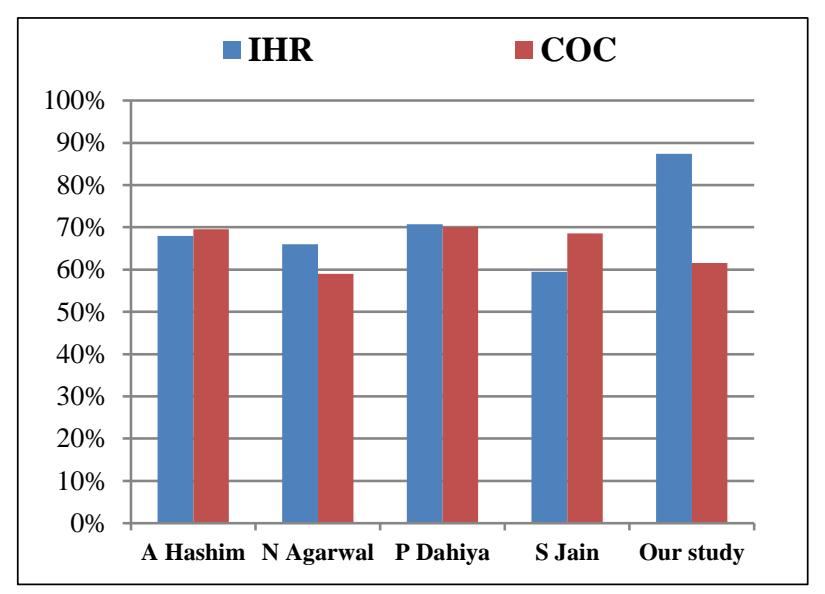

Figure 4: Comparison of percentage reduction in PBAC score in various studies. 
The patients had a sense of wellbeing at the end of the study due to significant decrease in the blood loss during the menstruation resulting in improvement in hemoglobin. Decrease in menstrual blood flow was comparable in both the groups. Percentage rise in hemoglobin was noted to be more and statistically significant in both groups in the present study, comparable to Hashim A et al, and Agarwal et al. ${ }^{13,14}$ The increase in hemoglobin levels was more in IHR group patients compared to COC users as observed by Agarwal et al. This may be explained by the superior cycle control achieved with the IHR. ${ }^{14}$

In the present study, the incidence of adverse events such as nausea, headache and mood swings was observed only in patients of COC group (4\%-8\%). Mastalgia was complained by 1 patient in IHR group and 3 patients in COC group but the difference was not statistically significant. The finding of a low incidence of these side effects compare favorably with other studies using the IHR versus COC for heavy menstrual bleeding. ${ }^{13-16}$ Mood swings/ depression were reported only in COC users (8\%); compared to none in IHR group and this difference was found to be statistically significant. Breakthrough bleeding also occurred less frequently in the IHR group than in the COC group. The lower dose of hormone producing steady serum level of steroid hormones with similar bioavailability of ethinyloestradiol in IHR users as compared to COC users has a beneficial role in reduction of systemic side-effect.

In contrast, 3 patients of IHR group reported of leucorrhoea compared to only 1 patient in the COC group. Our findings match those reported by other investigators. ${ }^{13-16}$ Two ring users reported ring expulsion. However, none discontinued treatment due to these ringrelated events. No coital dysfunction was reported in NuvaRing users. No significant changes in body weight were found in both groups as an effect of hormone therapy. However, the hemoglobin levels showed significant improvement in both groups. The current trial found IHR to be as effective as COC in treating HMB in carefully selected patients with an additional benefit of no requirement of daily intervention and lesser systemic side-effects.

\section{CONCLUSION}

Both the intravaginal hormonal ring (IHR) and the lowdose COC pills were effective short-term treatments of HMB with minimal side-effects. The IHR was better tolerated as the dose of $\mathrm{EE}$ was almost halved as compared to COCs. Superior cycle control, discreet use, once-a-month application, easy insertion and removal, lower incidence of breakthrough bleeding /spotting, less/no weight gain and non-interference with sexual activity led to greater patient compliance and satisfaction. Low incidence of side-effects compared favourably between both groups.

\section{ACKNOWLEDGMENTS}

Authors would like to thank all the faculty members and residents of the department of Obstetrics and Gynecology, Armed Forces Medical College, Pune and Command Hospital (Southern Command), Pune while conducting this study. Authors also acknowledge the patients who participated in the study.

Funding: No funding sources

Conflict of interest: None declared

Ethical approval: The study was approved by the Institutional Ethics Committee

\section{REFERENCES}

1. Munro MG, Critchley HO, Broder MS, Fraser IS. FIGO classification system (PALM-COEIN) for causes of abnormal menstrual bleeding in non-gravid women of reproductive age. FIGO Working Group on Menstrual Disorders. Int J Gynaecol Obstet. 2011;113:3-13.

2. National Collaborating Centre for Women's and Children's Health/National Institute for Health and Clinical Excellence. Heavy menstrual bleeding. NICE clinical guideline 44. London, UK: RCOG Press; 2007. Available at: https://www.rcog.org.uk/en/guidelines-researchservices/guidelines/heavy-menstrual-bleeding-niceclinical-guideline-44/. Accessed on $6^{\text {th }}$ January 2020.

3. Higham JM, O'Brien PMS, Shaw RW. Assessment of menstrual blood loss using a pictorial chart. Br J Obstet Gynaecol. 1990;97:734-98.

4. Janssen CA, Scholten PC, Heintz AP. A simple visual assessment technique to discriminate between menorrhagia and normal menstrual blood loss. Obstet Gynecol. 1995;85:977-82.

5. Lethaby A, Irvine G, Cameron I. Cyclical progestogens for heavy menstrual bleeding. Cochrane Database Syst Rev. 2008(1):CD001016.

6. Roumen FJME, Dieben TOM. Comparison of uterine concentrations of ethinylestradiol and etonogestrel - after use of a contraceptive vaginal ring and an oral contraceptive. Fertil Steril. 2006;85:57-62.

7. Roumen FJME. Review of the combined contraceptive vaginal ring, Nuvaring. Therap Clin Risk Manag. 2008;4(2):441-51.

8. Timmer CJ, Mulders TMT. Pharmacokinetics of etonogestrel and ethinylestradiol released from a combined contraceptive vaginal ring. Clin Pharmaco. 2000;39:233-42.

9. Milsom I, Lete I, Bjertnaes A. Effects on cycle control and body weight of the combined contraceptive ring, NuvaRing, versus an oral contraceptive containing 30 microg ethinyl estradiol and $3 \mathrm{mg}$ drospirenone. Hum Reprod. 2006;21:2304-11.

10. Roumen FJ. The contraceptive vaginal ring compared with the combined oral contraceptive pill: 
a comprehensive review of randomized controlled trials. Contracept. 2007;75:420-9.

11. Mulders TM, Dieben TO. Use of the novel combined contraceptive vaginal ring NuvaRing for ovulation inhibition. Fertil Steril. 2001;75:865-70.

12. Oddsson K, Leifels-Fischer B, Wiel-Masson D. Superior cycle control with a contraceptive vaginal ring compared with an oral contraceptive containing 30 microgethinylestradiol and 150 microg levonorgestrel: a randomized trial. Hum Reprod. 2005;20:557-62.

13. Abu Hashim $\mathrm{H}$, Alsherbini W, Bazeed $\mathrm{M}$. Contraceptive vaginal ring treatment of heavy menstrual bleeding: a randomized controlled trial with norethisterone. Contracept. 2012;85:246-52.

14. Agarwal N, Gupta M, Kriplani A, Bhatla N, Singh $\mathrm{N}$. Comparison of combined hormonal vaginal ring with ultralow-dose COC Pills in management menstrual bleeding: a pilot study (RCT). J Obs Gynae. 2016;36(1):71-5.
15. Dahiya P, Dalal M, Yadav A. Dahiya P, Dalal M, Yadav A, et al. Efficacy of combined hormonal vaginal ring in comparison to combined hormonal pills in heavy menstrual bleeding. Eur $\mathrm{J}$ Obstet Gynecol Reprod Biol. 2016;203:147-51.

16. Jain S, Vaid NB, Narang Y, Suneja A, Guleria K. A randomized controlled trial comparing the efficacy and side-effects of intravaginal ring (Nuvaring ${ }^{\circledR}$ ) with combined oral hormonal preparation in dysfunctional uterine bleeding. Journal of clinical and diagnostic research: JCDR. 2016;10(3):QC21.

Cite this article as: Saha T, Bharadwaj MK, Vardhan S. A randomized controlled trial to study the efficacy of intravaginal hormonal ring for control of heavy menstrual bleeding as compared to combined oral contraceptive pills. Int J Reprod Contracept Obstet Gynecol 2020;9:1205-12. 